\title{
Non-pecuniary Value of Employment and Natural Resource Extinction
}

\author{
Y. Hossein Farzin* \\ Department of Agricultural and Resource Economics \\ University of California, Davis, CA 95616, U.S.A. \\ farzin@primal.ucdavis.edu.
}

\author{
Ken-Ichi Akao \\ School of Social Sciences \\ Waseda University, Tokyo, Japan \\ Akao@waseda.jp
}

November 2005

\begin{abstract}
$\underline{\text { Abstract }}$
We assume that people value employment not only to earn income to satisfy their consumption needs but also as a means of community/social involvement that provides socio-psychological (non-pecuniary) benefits. We show that the latter incentive can encourage full employment harvesting resources and explain why poor resource-based communities may exhaust a natural resource in a finite time even if there is a sustainable path of resource consumption available. We show that communities could sustain their natural resources by using outside-the-community employment and economic diversification, but, to be effective, such policies must ensure that the outside wage rate and the initial capital stock are above certain minimum levels, which will be higher the longer these policies are delayed.
\end{abstract}

*Corresponding author.

Key Words: Non-pecuniary effects, employment value, resource extinction, sustainability JEL Codes: E24, O12, O13, Q28

Copyright 2005 by Farzin and Akao. All rights reserved. Readers may make verbatim copies of this document for non-commercial purposes by any means, provided that this copyright notice appears on all such copies. 


\section{Non-pecuniary Value of Employment and Natural Resource Extinction}

\section{Introduction}

Ecologists often argue that the extinction of natural resources is a consequence of our rather self-centered behavior and partial view of the ecosystem. In order to satisfy human needs, many people behave as if they are independent of the ecosystem that provides the basic materials to sustain life, with materialistic needs causing the problem.

In this paper we take a different view. We argue that in closed, resource-based communities, particularly those in developing nations, the satisfaction of non-materialistic needs, or, more specifically, the psychological and sociological benefits of being employed, may explain the extinction of natural resources. This can occur if employment is both a source of earning income to satisfy materialistic needs as well as the principal means of community involvement or a social norm. We go beyond the standard neoclassical income-leisure choice, which considers nonwork time as leisure and values it by the cost of income foregone. We follow sociologists and psychologists (see, for example, Whelan (1994) and Agerbo et al (1997)), who recognize that, in addition to being a source of income and material satisfaction, employment can provide non-materialistic satisfaction. The socio-psychological benefits of employment can be particularly strong in poor, rural resource-based (RRB) communities where working is a social custom or norm and an important constituent of one's identity. In such communities being unemployed is a deviation from the social norm, entailing loss of utility.

Allowing for the non-pecuniary benefits of employment can give rise to "full employment obsession" in such resource-dependent communities. If labor is the principal input (or "effort") that determines the rate of resource harvest, a "full employment obsession" can explain why it may be optimal to extinct a resource in a finite time even if an alternative sustainable path is available. However, sustainability may require the community to restrict employment level below its "full" level for a transitory period to allow the resource stock to grow. Thus, we offer a "full-employment" alternative explanation to the ecologists "selfish" explanation for exhaustion or extinction of natural resources. Even if communities recognize the ecological constraints on regenerative capacity of the resource, the negative socio-psychological effects of unemployment on individuals' subjective well-being may make it too hard for RRB communities to tolerate unemployment to let the resource stock regenerate. In such situations, employment outside the resource sector and economic diversification may satisfy both resource sustainability and full employment goals.

Economics literature provides few studies of conditions for the optimal extinction of a natural resource in a finite time. Clark (1973) and Spence (1973), the classic papers, showed that when there is purely an economic motivation (specifically, maximization of the present value of the stream of profits from resource sales), if the resource owner's discount rate is 
higher than the growth potential (intrinsic/biological rate of growth) of the resource, then it is economically optimal to deplete the resource stock to extinction in a finite time. Spence (1973) corroborated this result numerically in the case of the blue whale, which was then an endangered species ${ }^{1}$. Brander and Taylor (1998) analyzed the dynamic interactions of an open-access renewable resource and people to explain the collapse of the Easter Island civilization, showing that the resource and population dynamics had an oscillatory behavior, and that a change in parameters, reflecting climatic changes on the island, allowed the population or the resource stock to fall below critical levels for regeneration, leading to the extinction of the population or the resource. The key feature distinguishing our paper is the explanation for an excessive supply of effort (labor) because of the socio-psychological effects of employment as asocial norm on the well-being of resource harvesters in a RRB community.

The paper proceeds as follows. Section 2 briefly reviews some of the empirical evidence for non-pecuniary value of employment and discusses why it may be particularly important in RRB communities. Section 3 uses the standard bionomic model of a renewable resource to show the incompatibility of full employment with resource sustainability when non-pecuniary benefits of employment are strong, and identifies the condition under which resource extinction in finite time is optimal. Section 4 explores the possibilities of outside employment and economic diversification as potential options to achieve both full employment and resource sustainability, and shows that for such options to be effective the outside wage rate and the initial capital stock should exceed certain minimum levels. Section 5 concludes.

\section{Non-pecuniary Value of Employment}

Employment is a principal means for people to connect and become involved with communal activities. In turn, community involvement directly or indirectly brings the individual a sense of self-esteem, self-respect, belonging, identity, recognition, reputation, friendship, security, and status, all of which are ingredients of one's quality of life and

\footnotetext{
1 Note that the well-known case of extinction of a population under the open-access regime (sometimes known, incorrectly, also as the "tragedy of commons") may be viewed as a special case of this result when the discount rate approaches infinity. However, Cropper and Lee (1979) show that this result need not hold when the harvest price is allowed to be inversely related to the harvest rate rather than assumed to be constant, as in Clark (1973). In that case, the result holds only for sufficiently small initial stocks but not for sufficiently large initial stocks even if the discount rate is infinite (see also Farzin (1984) for the role of the initial stock size for the effect of the discount rate on resource depletion). In a related paper, Smith (1975) explains the mass extinctions of mega fauna in America during the late Pleistocene by using an open-access resource (free-access hunting) model. He shows that if the average biological growth rate at zero stock level is low and less than the equilibrium harvest per unit biomass, the extinction of a species will occur due to hunting pressure.
} 
satisfaction (Jahoda (1988)(1982)(1981)). ${ }^{2}$ Being employed is an essential determinant of happiness; the unemployed have significantly lower well-being scores in the social psychological and labor economics literature (see, for example, Fryer and Payne (1986), Feather (1990) and Argyle (2001)). Further, Lucas, et al (2004), find strong statistical evidence that the adverse effect of unemployment on individuals' subjective well-being persists even after they become employed again. More interestingly, empirical work in labor economics literature has established that unemployment is strongly negatively correlated with individual well-being, even after controlling for income and other individual characteristics. In other words, the unemployed are generally worse off than the employed, and by more than their lower income would predict (see, for example, Clark and Oswald (1994), Korpi (1997), Winkelmann and Winkelmann (1998), Di Tella, MacCulloch and Oswald (2001)(2003), and Clark (2003) and references cited therein). For example, Winkelmann and Winkelmann (1998) used panel data on life satisfaction from German-Socio-Economic Panel for 1984-1989, where the individual's subjective well-being was measured on an ordinal scale from 0 to 10 . After controlling for income and various observed individual characteristics and specific fixed effects, they found that (a) being unemployed has a significant and substantial negative effect on satisfaction, and (b) the pecuniary costs of unemployment, occurring directly through reduced income, are much smaller than the non-pecuniary costs, occurring indirectly through reduced well-being. They estimated that income would have needed to be increased by a factor of seven in order to generate an increase in satisfaction large enough to offset the adverse effect of unemployment. Di Tella, MacCulloch and Oswald (2001) came to similar conclusions. Using the Euro-Barometer data on individual life satisfaction, ordered on a four-point scale for 12 European countries for the period 1975-91, they found that, controlling for the income loss and other indirect effects, being unemployed has a strong negative effect on well-being. Based on Di Tella et. al's estimated happiness function, Frey and Stutzer (2002) calculated the compensation variation for being unemployed rather than holding a job and noted that "a move from the lowest income quartile to the highest income quartile would not be enough to offset the adverse effect of unemployment, suggesting that unemployed people suffer high non-pecuniary costs (p.402)". These results suggest that being unemployed significantly reduces people's well-being, even when receiving the same income as when employed. As Di Tella, MacCulloch and Oswald (2003, pp. 819-820) note "Being unemployed is much worse than is implied by the drop in income alone. The economist's standard method of judging the disutility from being laid off focuses on pecuniary losses. According to our calculations, that is

\footnotetext{
${ }^{2}$ For a general and insightful treatment of the effects of identity on economic behavior and outcomes, see Akerlof (2000). Also, see Akerlof and Kranton (2003)(2005) for an analysis of the effects of workers' identity on incentives to exert effort, wage rate variation, and firms' optimal management strategies. For a survey of the social psychological consequences of unemployment and implications for behavioral macroeconomic model and policy see Darity and Goldsmith (1996).
} 
a mistake, because it understates the full well-being costs, which according to the data, appear to be predominantly nonpecuniary." The facts that in advanced industrial countries, where social safety nets cushion joblessness, some individuals prefer to be employed with earnings less than the unemployment benefit or to engage in voluntary work are evidence of non-pecuniary value of employment. ${ }^{3}$ Furthermore, the presence of non-pecuniary value of employment is strongly suggested by Mulligan's (1998) insightful empirical study of the dramatic increase in civilian work in the United States during World War II. Ruling out the changes in workers' budget sets (the after-tax real wages were substantially lower than either before or after the war) or other pecuniary explanations such as wealth effects of government policies, wage-induced intertemporal substitution, and changes in the nonmarket price of time, she concluded that non-pecuniary motives such as patriotism and changing discrimination against women can explain the phenomenon.

Finally, using data from the Alaska salmon fisheries, which have been subject to entry limitations since 1975, Karpoff (1985) tested the hypothesis that fishermen receive significant non-pecuniary benefits by estimating the present value of the expected rent stream from fishing and examining whether permit prices reflected a premium above what monetary income alone would suggest. He found that the continued presence of many low-income fishermen in the fisheries was evidence that they, at least, derive non-pecuniary benefits from fishing.

The negative socio-psychological effects of unemployment on well-being are likely to be particularly strong in closed RRB communities, when compared, for example, with the effects on working populations in cosmopolitan urban areas. The reason is that in such communities, being productive is often a social norm or custom, giving the individual a sense of belonging and identity. Being unemployed is considered a deviation from the norm, and carries with it significant negative psychological and sociological effects, which every community member tries to avoid. ${ }^{4}$ This is less true in urban areas with diversified economies that are open to trade with outside, and where the role of the individual is less apparent to the rest of society. As noted by Veblen (1899, P.69 and P.75) long ago, in urban areas individuals are often recognized for their consumption behavior, so that "distinction in consumption" or "conspicuous consumption" is a means of gaining recognition. It seems therefore reasonable to suppose that the social norm of work can be especially strong in closed RRB communities; that is, a large portion of their active populations adhere to it. Interestingly, in a revealing empirical study of the psychological effect of unemployment on individual well-being, Clark (2003)

\footnotetext{
${ }^{3}$ Moffitt (1983) found strong empirical support for the existence of welfare stigma among eligible but non-participants in AFDC-U, the welfare program for which families with an unemployed male were eligible.

${ }^{4}$ In many RRB communities, especially in developing countries, sharing the efforts needed for resource harvesting is a social norm which in turn stems from the community norm of sharing the harvest. Diverging from the norm of sharing efforts can inflict social shame and cause one to be ostracized.
} 
finds a strong and statistically significant positive correlation between the negative psychological effect of unemployment and the degree of adherence to the social norm of employment. Accordingly, being unemployed is likely to be particularly stigmatic in RRB communities.

\section{Full-Employment Obsession and Resource Extinction}

\subsection{Sufficient Condition for Extinction in finite time}

Dasgupta and Maler (1995, P. 2373) emphasize that "poor countries are for the most part biomass-based subsistence economies, in that their rural folk eke out a living from products obtained directly from plants and animals." Many rural communities in poor countries depend on natural resources, so it may be thought that residents of such communities would have sustainability as a major social and economic goal. However, if the non-pecuniary (socio-psychological) value of employment is strong, full employment can become an equally important goal. Achieving sustainability and full employment simultaneously is not a problem if the community's initial resource base is sufficiently large and the resource regenerative capacity is sufficiently high; if not, there may be a conflict between sustainability and full employment. This section identifies the precise conditions that make extinction of the resource in a finite time socially optimal.

Economics literature traces sustainable resource allocation to the so-called Ramsey (1928) problem: seeking an optimal path along which the resource stock (in his case, manufactured capital) stock converged to some positive level, (i.e., an interior optimal stationary state) regardless of the initial size of the stock. In our case, an interior optimal stationary state is a sustainable stock level. However, if the optimal policy is full employment, the resource may become extinct.

An optimal path that leads to resource extinction in finite time requires a marginal productivity of the resource stock that is less than the time discount rate. (see, for example, Clark (1973) and Spence (1973)). In our case, the optimality of extinction occurs regardless of the time discount rate, and we show that under certain conditions full employment until resource extinction is socially optimal (full employment obsession). ${ }^{5}$

Consider an RRB community with a constant population (labor force) size of $\bar{E}$. The community owns and lives off of a stock of a single renewable resource (e.g., a fishery or

\footnotetext{
${ }^{5}$ It has been well known that in the game theoretic framework the resource extraction towards finite time extinction can occur as a result of "individual" rationality under open-access regime. Gordon (1954) and Hardin's (1968) influential paper, "the tragedy of commons", are among the early works alluding to this, followed by a number of rigorous analyses including Dasgupta (1982), Cornes and Sandler (1983)(1996), and Sorger (1998), among others. It should be emphasized, however, that in the present study there is no open-access to the resource and no strategic interactions among the community members who are assumed to fully and collectively own the resource.
} 
forest) the size of which is denoted by $S(t) \geq 0$ at time $t$. The change in the resource stock over time is described by

$$
\dot{S}(t)=G[S(t)]-H[E(t), S(t)] \quad \forall t \geq 0,
$$

where $G(S)$ is the natural growth function, $H(E, S)$ is the harvest function where $0 \leq E(t) \leq \bar{E}$ is the level of "effort", which we simply assume to consist only of the community labor force at time $t$. We make the following assumptions:

(A1): $G(S)$ is concave in $S$ (i.e., $G_{S S} \leq 0$ ) and there is a unique biological carrying capacity $k>0$ such that $G(k)=0, G(S)>0$ for all $0<S<k$, and $G(0)=0$.

(A2): $H(E, S)$ is an increasing function and strictly concave in $E$ (i.e. $\left.H_{E}>0, H_{S}>0, H_{E E}<0\right)$.

(A3): For any given $S_{0} \geq 0$, the full-employment harvest rate $H(\bar{E}, S(t))$ exceeds the population growth $G(S(t))$, so that $\dot{S}(t)=G[S(t)]-H[\bar{E}, S(t)]<0$, thus permitting finite time exhaustion of the resource stock. Notice that this assumption can hold if the resource growth rate is sufficiently low relative to the stock effect on harvest $\left(H_{S}(\bar{E}, S)\right)$, in which case the curve of harvest function at full employment, $H(\bar{E}, S)$, may everywhere lie above that of population growth function, $G(S)$.

Assumptions (A1) and (A2) are quite general. On the other hand, assumption (A3) reflects the situation when full employment and sustainable resource use are incompatible. Our objective is to show under what condition resource exhaustion in a finite time is socially optimal.

Denote by $W(C, E)$ the community's instantaneous social welfare function ${ }^{6}$, where $C \geq 0$ and $E \geq 0$ are the aggregate consumption and employment. We assume that:

(A4): $W(C, E)$ is jointly concave in $C$ and $E, W_{C}>0$, and $W_{E}>(<) 0$ if $E<(>) \hat{E}(C)$, where $\hat{E}(C)$ is the critical level of employment for which $W_{E}(C, \hat{E})=0$ and satisfies $\hat{E}^{\prime}(C)<0$.

This social welfare function can be derived consistently from an extended individual utility function $U=U(c, z, m)$ where $c \geq 0$ is consumption, $z=T-l \geq 0$ is leisure time, which is equal to the fixed total time endowment $T$ less labor time $l \geq 0$, and $m(l) \geq 0$ captures the non-pecuniary effects (NPE) of employment, which is assumed to increase with one's

${ }^{6}$ The time arguments of the functions are omitted whenever no confusion arises. 
community involvement and hence labor time (i.e. $m^{\prime}(l)>0$ ). The utility function is assumed to satisfy the usual assumptions that it is increasing in each of its arguments (i.e., $U_{c}>0, U_{z}>0, U_{m}>0, U_{c} \rightarrow \infty$ as $c \rightarrow 0$ ) at decreasing rates (i.e., $U_{c c}<0, U_{z z}<0, U_{m m}<0$ ) and that both leisure and NPE are complements with consumption (i.e., $\left.U_{c z}>0, U_{c m}>0\right)^{7}$. One can then rewrite $U$ in the reduced form of $u(c, l)=U(c, T-l, m(l))$ so that the overall, or net, marginal utility of labor time is $\partial u / \partial l=d U / d l=-U_{z}+U_{m} m^{\prime}(l)$, where the first term on the right-hand side is the marginal disutility of labor and the second term is the marginal utility of labor that arises from the non- pecuniary value of employment. We assume, as is plausible, that, at any given consumption level $c$, for sufficiently large values of $l$ the first term dominates the second one (implying that at the margin labor becomes a net source of disutility, $\left.u_{\ell}(c, l) \equiv \partial u / \partial l<0\right)$ and vice versa for sufficiently small $l$, so that for each $c>0$, there exists a unique $\hat{l}(c)>0$ such that $\partial u(c, l) / \partial l=-U_{z}+U_{m} m^{\prime}(l)>(\leq) 0$ as $l<(\geq) \hat{l}(c)$. That is, $\hat{l}(c)$ is the threshold labor time, at consumption level $c$, so that the marginal disutility of labor exactly offsets its marginal utility.

As shown in Farzin and Akao (2004a), at sufficiently low wage (and hence consumption) rates, leisure may lose its desirability to such an extent (i.e., $U_{z}$ becomes so small) that work can even become a source of utility (i.e., $\partial u(c, l) / \partial l>0)$, so that one chooses to work as much as possible $(l=T)$. The social welfare function $W(C, E)$ inherits these properties. Specifically, employment itself enhances the community's well-being if its level is below the critical level $\hat{E}$, or if, for any given employment level, the consumption level is too low. In fact, the direct link between the two functions becomes obvious if we adopt the concept of utilitarian social welfare and assume that the community's population $\bar{E}$ consists of identical individuals. In that case, we have $W(C, E)=\bar{E} u(C / \bar{E}, E / \bar{E})=\bar{E} u(c, l)$ and $\hat{E}(C)=\bar{E} \cdot \hat{l}(C / \bar{E})=\bar{E} \cdot \hat{l}(c)$.

Denote by $\rho$ the time discount rate. The social planner's problem is:

$$
\begin{aligned}
& \max _{E(t)} \int_{0}^{\infty} W[H(E, S), E] e^{-\rho t} d t \\
& \text { subject to } \quad \dot{S}(t)=G[S(t)]-H[E(t), S(t)] \text {, } \\
& 0 \leq E(t) \leq \bar{E}, 0 \leq S(t), \quad \forall t \geq 0 ; \\
& S(0)=S_{0} \in[0, k] \text { given. }
\end{aligned}
$$

Define $w(E, S) \equiv W[H(E, S), E]$, i.e. $w$ is a reduced form of the social welfare

\footnotetext{
${ }^{7}$ For more details about the properties of the extended individual utility function, see Farzin and Akao (2004a)(2004b).
} 
function $W$, and satisfies $w_{S S}<0$. Then, the Hamiltonian of problem (2) is:

$$
\Psi(E, S, \lambda)=w(E, S)+\lambda[G(S)-H(E, S)]
$$

where $\lambda=\frac{\partial \Psi}{\partial S}$ is the co-state variable and measures the shadow price of the resource stock. The necessary conditions for a solution of problem (2), $\left(E^{*}(t), S^{*}(t)\right)$, are that, at each $t \geq 0$ :

$$
\Psi\left[E^{*}(t), S^{*}(t), \lambda(t)\right]=\max \left[\Psi\left(E(t), S^{*}(t), \lambda(t)\right) \mid E(t) \in[0, \bar{E}]\right],
$$

and

$$
\dot{\lambda}(t)=\rho \lambda(t)-\partial \Psi\left[E^{*}(t), S^{*}(t), \lambda(t)\right] / \partial S .
$$

Denoting by $\tilde{E}(S, \lambda)$ the implicit function solving $\Psi_{E}(\tilde{E}(S, \lambda), S, \lambda)=0$, we refer to $\tilde{E}(S, \lambda)$ as the stationary point of $\Psi$. It is worth noting that the maximum condition (4) does not necessarily hold at the associated stationary point $E=\tilde{E}\left(S^{*}(t), \lambda(t)\right)$, implying that the employment constraint may be binding (i.e., $E(t)=\bar{E}$, or $0 \forall t \geq 0$ ) at the optimum. This is because the Hamiltonian as a function of $E$ may not be concave at the stationary point, due to the concavity of the harvest function $H(E, S)$ in $E$. When the maximum condition holds with $E(t)=\bar{E}$, it indicates that the community gives priority to full employment rather than to sustainability. The following proposition identifies the precise conditions under which this occurs.

Proposition1: Assuming that problem (2) has a solution, if

$$
\frac{-\tilde{E} H_{E E}(\tilde{E}, S)}{H_{E}(\tilde{E}, S)}-\frac{-\tilde{E} w_{E E}(\tilde{E}, S)}{w_{E}(\tilde{E}, S)}>0
$$

the optimal path is the full-employment obsession path.

Proof: See Appendix A1 for the proof.

The economic interpretation for Proposition 1 is straightforward once we note from (3) that a small increase in employment level $E$ has two beneficial effects on the current social welfare: by increasing the harvest and hence consumption level and through its non-pecuniary effect $\left(w_{E}=W_{H} H_{E}+W_{E}\right)$. But, by raising the harvest level, it also lowers the resource stock, which involves a cost amounting to $\lambda H_{E}(E, S)$ in utility terms. Both the marginal benefit $\left(w_{E}\right)$ and cost $\left(\lambda H_{E}\right)$ of employment decline as employment level is increased. Accordingly, Proposition 1 states that full employment obsession, and thus finite time extinction of the resource, occurs when the (employment) elasticity of the marginal cost of employment (the first term of the LHS of (6)) always exceeds the elasticity of its marginal benefit (the second term). In other words, in response to a given percentage increase in employment level, the 
latter declines proportionally less than the former does, implying that the marginal net benefit from employment will always be increasing with employment level. We shall refer to condition (6) as condition for full employment obsession. ${ }^{8}$

Two important points follow from this condition. First, it neither involves the natural growth function $G(S)$ nor the time discount rate $\rho$, respectively because of assumption (A3) and the fact that along the optimal path the harvest rate at any time is at its maximum feasible, i.e. at the full-employment, level, $H(\bar{E}, S)$, so that a change in the discount rate has no effect on the optimal harvest path. Therefore, the mechanism causing the finite time resource extinction in our model differs sharply from the ones noted in the literature. Here it is the desirability of employment as an independent determinant of well-being that drives the economy to the full-employment path and leads to resource extinction in finite time. Second, we note that condition (6) cannot hold if $W_{E}(C, E) \leq 0$ and $W_{C E}(C, E) \leq 0 .{ }^{9}$ But, $W_{E}(C, E)<0$ is the basic premise of the standard labor supply theory that regards labor only as source of disutility and ignores the positive socio-psychological effect of employment on well-being, and if it bears any truth at all, it should be about the preferences of urban population in rich industrialized societies. This suggests that the condition for full employment obsession is more likely to hold for preferences of the poor people in RRB communities, where one expects $W_{E}(C, E)>0 .^{10}$

\subsection{A Specific Illustration}

In this subsection, we present an example illustrating the full-employment obsession path as the optimal path. Let us specify the functions in Problem (2) as follows:

(1) The natural growth function takes the Logistic form:

$$
G(S)=r S\left(1-\frac{S}{k}\right), r>\rho, k>0
$$

The natural (intrinsic) growth rate, $r$, is the resource's maximum potential reproductive rate. So,

${ }^{8}$ Technically, the condition requires that at the stationary point $\tilde{E}$, the harvest function $H$ be more concave in $E$ than the welfare function $w$ is, implying that $\frac{d}{d E}\left(\frac{{ }_{E}}{H_{E}}\right)>0$ at that point.

9 To see this formally, recall that $w(E, S) \equiv W[H(E, S), E]=W(C, E)$. Differentiate (3) with respect to $E$ to have $W_{C} H_{E}=-W_{E}+\lambda H_{E}$, and use this to obtain

$$
\begin{aligned}
& \frac{-E H_{E E}}{H_{E}}-\frac{-E w_{E E}}{w_{E}}=\frac{-E H_{E E}}{H_{E}}-\frac{-E\left(W_{C C}\left(H_{E}\right)^{2}+2 W_{C E} H_{E}+W_{C} H_{E E}+W_{E E}\right)}{\lambda H_{E}} \\
& =\frac{-E H_{E E}}{H_{E}}\left(1-\frac{W_{C}}{\lambda}\right)-\frac{-E\left(W_{C C}\left(H_{E}\right)^{2}+2 W_{C E} H_{E}+W_{E E}\right)}{\lambda H_{E}}==\frac{-E H_{E E}}{H_{E}}\left(\frac{W_{E}}{\lambda H_{E}}\right)-\frac{-E\left(W_{C C}\left(H_{E}\right)^{2}+2 W_{C E} H_{E}+W_{E E}\right)}{\lambda H_{E}}<0,
\end{aligned}
$$

where all functions are evaluated at $(\tilde{E}(S, \lambda), S, \lambda)$.

${ }^{10}$ It is also worth noting that condition (6) does not hold if the harvest function is convex or linear in $E$ (i.e., if $H_{E E} \geq 0$ ), although the latter has been commonly assumed in the literature. 
the inequality $r>\rho$ implies that the resource is economically productive, thus further sharpening the distinction between our model and the previous studies of the condition for finite time resource extinction.

(2) The harvest function has a Cobb-Douglas form:

$$
H(E, S)=\gamma E^{\alpha} S^{\beta}, \gamma, \alpha, \beta>0, \alpha+\beta \leq 1 ;
$$

Where it is noted that the harvest is bounded above by $\bar{H}=H(\bar{E}, k)$. The necessary condition for finite time extinction of the resource is $\lim _{S \rightarrow 0} H_{S}(\bar{E}, S)=\infty .{ }^{11}$ The Cobb-Douglas harvest technology (8) does have this property. By assuming that the total productivity is high enough to satisfy

$$
\gamma>\frac{r[(1-\beta) K]^{1-\beta}}{(\bar{E})^{\alpha}(2-\beta)^{2-\beta}},
$$

assumption (A3) holds, implying that the resource is exhausted in a finite time. (See Appendix A2 for the proof.)

(3) The social welfare function also takes a simple Cobb-Douglas form:

$$
W(C, E)=C^{\eta_{C}} E^{\eta_{E}}, \eta_{C}, \eta_{E}>0, \eta_{C}+\eta_{E}<1 .
$$

It is concave, increasing in consumption and employment, and $W(0, E)=W(C, 0)=0$ bounded below. Further, it satisfies $W_{E}>0$ and $W_{C E}>0$. Especially because of the latter two properties, the Cobb-Douglas social welfare function suits our purpose particularly well. For, in view of the discussion in Section 3, it can be attributed to preferences of an RRB community so acutely poor that a rise in employment level always has a positive effect on the community's well-being and its marginal value of consumption; that is, for all feasible paths of resource use $(C, E)$, $W_{E}>0$ and $W_{C E}>0$ never switch signs. ${ }^{12}$

The reduced form of the social welfare function is

$$
w(E, S)=W[H(E, S), E]=\gamma^{\eta_{C}} E^{\alpha \eta_{C}+\eta_{E}} S^{\beta \eta_{C}} .
$$

Since

\footnotetext{
${ }^{11}$ To see this, suppose that $\lim _{S \rightarrow 0} H_{S}(\bar{E}, S)=\xi<\infty$. Then, we can approximate the harvest function with $\xi S$ and the natural growth function with $r S$ in a neighborhood of the origin. Thus, $\dot{S}=G(S)-H(E, S) \approx S(r-\xi)$. With a linear differential equation, it takes infinite time to exhaust the resource.

12 Notice that the boundedness condition, $W(0, E)=0$, is necessary to have an optimal path with finite time extinction. On the other hand, the condition $\lim _{c \rightarrow 0} W_{C}(C, E)=\infty$ defies the optimality of resource extinction in finite time, without non-pecuniary effects of employment.
} 


$$
\frac{-E H_{E E}}{H_{E}}-\frac{-E w_{E E}}{w_{E}}=(1-\alpha)-\left(1-\alpha \eta_{C}-\eta_{E}\right)=\eta_{E}+\alpha \eta_{C}-\alpha
$$

the condition for full employment obsession (6) simplifies to ${ }^{13}$

$$
\left(\eta_{E}+\alpha \eta_{C}\right)>\alpha
$$

That is, for this specific case, the net marginal welfare effect of employment will always be positive if the elasticity of social welfare level with respect to employment $\left(\eta_{E}+\alpha \eta_{C}\right)$ exceeds the elasticity of the harvest level, $\alpha .{ }^{14}$

\section{Policy Options for Sustainability}

In this section, we explore some economic policies that can induce an RRB community to adopt a sustainable path of resource use. We do this in the context of the specific functional forms (7)-(9) used in the previous section. We assume that before a policy measure is implemented the condition $\left(\eta_{E}+\alpha \eta_{c}\right)>\alpha$ holds, so that the optimal path is that of full employment obsession with resource exhaustion in finite time.

It is worth noting that this condition consists of two elasticities of the welfare function, $\eta_{E}$ and $\eta_{C}$, and one elasticity of the harvest function, $\alpha$. Thus, to have a potential to prevent the finite time resource extinction, two broad groups of policy measures may be distinguished. One group consists of policies that can affect the community's preferences and/or its harvesting technology in such a way that at least one of the three elasticities is suitably changed so as to defy the condition $\left(\eta_{E}+\alpha \eta_{c}\right)>\alpha$. By itself, this requirement rules out a number of policy options as ineffective. For example, it is easy to show that any economic assistance to the community in the form of a commodity grant or subsidy that proportionally

\footnotetext{
${ }^{13}$ For the proof of the existence of an optimal path see Appendix A3.

${ }^{14}$ Notice, again, from (11) and $\eta_{c}<1$ that the full-employment obsession, and hence the finite time extinction, can occur only if employment also directly enhances the individual's utility and therefore the community's welfare, i.e., only if $\eta_{E}>0$.
} 
augments consumption will not help to prevent resource extinction. ${ }^{15}$ Similarly, unless it sufficiently reduces $\alpha$, a technological assistance that is confined to resource harvesting may not only fail to induce resource use sustainability, it can even worsen the situation because it could accelerate the pace of resource exhaustion. Examples of such technologies are those of more efficient but "effort-neutral" or "effort-augmenting" harvesting technologies, and, perhaps ironically, may also include the technologies that enhance the resource stock (for example, radar-based fishing vessels capable of locating fish stocks by radar). The other group of policies are those that take the community's preferences and harvesting technology as given, at least in the short run, and resort to formal labor markets as mechanisms to absorb what would otherwise constitute excessive effort (from the sustainability viewpoint) in the resource sector.

In what follows we do not explore potential policies in the first group as they would involve changing consumption habits (preferences)-much in accord with ecologists' advicewith the effect to lower the value of $\eta_{C}$, or changing the community's attitude towards employment (social norm of work), with the effect to lower the value of $\eta_{E}$, or both. Such policies would aim to encourage changes in cultural values. Accordingly, besides involving economic incentives, they involve complex and community-specific social and political incentives whose effects may take a long time to evolve, perhaps longer than the resource can survive. Instead, we concentrate on two economic policy options in the second group and derive conditions under which they can lead the community to a full employment and yet sustainable path of resource use. The main difference between the two options is that one of them considers employment opportunities outside the community while the other aims at additional employment opportunities through economic diversification.

15 It can also be shown that when economic assistance to the community is in the form of a real flow of lump-sum transfers or foreign grants (denoted by $X(t) \geq 0, \forall t \geq 0$ ), one has

$\frac{-E H_{E E}}{H_{E}}-\frac{-E w_{E E}^{X}}{w_{E}}=\eta_{E}-\alpha+\alpha \eta_{c} \varphi\left(1+\frac{X}{H}\right)$,

where $w^{X}(E, S, t)=W[H(E, S)+X, E]=[H(E, S)+X]^{\eta_{c}} E^{\eta_{E}}$ and $\quad$ the function $\quad \varphi \quad$ is defined by $\varphi(x) \triangleq \frac{\left(\alpha+\eta_{E}\right) x-\alpha\left(1-\eta_{C}\right)}{x\left(\alpha \eta_{C}+\eta_{E} x\right)}, \quad x=\left(1+\frac{X}{H}\right) \geq 1$. Since $\lim _{x \rightarrow \infty} \varphi(x)=0$, it follows that such policies can be effective $\left(\eta_{E}-\alpha+\alpha \eta_{c} \varphi\left(1+\frac{X}{H}\right) \leq 0\right)$ if the rate of transfer relative to harvest rate is any time sufficiently large and if $\eta_{E}<\alpha$. However, if $\eta_{E} \geq \alpha$, the full employment obsession path is optimal and resource extinction occurs in finite time, no matter how large the flow of transfers. 


\subsection{Outside Employment Opportunity}

Suppose that an outside labor market is opened to our RRB community. Denote by $\omega>0$ the constant wage rate in units of harvest. ${ }^{16}$ Notice that any optimal path is a full employment path because now people can work without necessarily reducing the resource stock. Thus, the social planner's problem is:

$$
\begin{aligned}
& \max _{E(t)} \int_{0}^{\infty} W[H(E(t), S(t))+\omega(\bar{E}-E(t)), \bar{E}] e^{-\rho t} d t, \\
& \text { subject to } \dot{S}(t)=G[S(t)]-H[E(t), S(t)], \\
& \quad 0 \leq E(t) \leq \bar{E}, 0 \leq S(t), \text { for each } t \geq 0 ; \\
& \quad S(0)=S_{0} \geq 0 \text { given. }
\end{aligned}
$$

The Hamiltonian is

$$
\Psi(E, S, \lambda)=w^{O}(E, S)+\lambda[G(S)-H(E, S)]
$$

where $w^{O}(E, S)$ denotes the welfare function with outside employment opportunity, and is given by

$$
w^{O}(E, S)=[H(E, S)+\omega(\bar{E}-E)]^{\eta_{C}} \bar{E}^{\eta_{E}} .
$$

Assuming the existence of an optimal path and denoting it by $\left(E^{*}(t), S^{*}(t)\right)$, if $\bar{E} \geq E^{*}(t)>0$, we have

$$
\Psi_{E}\left(E^{*}, S^{*}, \lambda\right)=\eta_{C}\left[H\left(E^{*}, S^{*}\right)+\omega\left(\bar{E}-E^{*}\right)\right]^{\eta_{C}-1}\left[H_{E}\left(E^{*}, S^{*}\right)-\omega\right] \bar{E}^{\eta_{E}}-\lambda H_{E}\left(E^{*}, S^{*}\right) \geq 0
$$

which implies ${ }^{17}$

$$
H_{E}\left(E^{*}, S^{*}\right)-\omega>0
$$

or, by substituting for $H_{E}\left(E^{*}, S^{*}\right)=\alpha \gamma\left(E^{*}\right)^{\alpha-1} S^{* \beta}$,

$$
E^{*}<\left(\frac{\alpha \gamma\left(S^{*}\right)^{\beta}}{\omega}\right)^{\frac{1}{1-\alpha}} .
$$

This sets an upper bound on employment in the resource sector $E^{*}$. Since

\footnotetext{
${ }^{16}$ The assumption of constant real wage rate is made for expositional simplicity. The results that follow also hold for time dependent wage rates $\omega(t)$.

${ }^{17}$ Notice that because of the marginal social cost of employment due to the negative stock effect of increased harvest rate (i.e., $-\lambda H_{E}$ ), the optimal employment allocation requires that the marginal product of employment in the resource sector to exceed the outside wage rate .
} 
$\dot{S}^{*}=G\left(S^{*}\right)-H\left(E^{*}, S^{*}\right)$, an optimal path $S^{*}(t)$ satisfies

$$
\dot{S}^{*}>G\left(S^{*}\right)-H\left[\left(\alpha \gamma S^{* \beta} / \omega\right)^{\frac{1}{1-\alpha}}, S^{*}\right]
$$

Let $\omega(S)$ be the function that solves $G(S)-H\left[\left(\alpha \gamma S^{\beta} / \omega(S)\right)^{\frac{1}{1-\alpha}}, S\right]=0$, that is

$$
\omega(S)=\left[\frac{\gamma \alpha^{\alpha} S^{\beta}}{G(S)^{1-\alpha}}\right]^{1 / \alpha}
$$

(Note in particular that for $\omega\left(S_{0}\right), G\left(S_{0}\right)-H\left[\left(\alpha \gamma S_{0}{ }^{\beta} / \omega\left(S_{0}\right)\right)^{\frac{1}{1-\alpha}}, S\right]=0$.)

Then, we have

Proposition 2: If $\omega \geq \omega\left(S_{0}\right)$, then $S^{*}(t) \geq S_{0}$, i.e. along an optimal path the resource stock never declines below the initial stock level.

Proof: By (17) and (16), if $S^{*}(t)=S_{0}$, then $\dot{S}^{*}(t) \geq 0$.

Thus, as long as the outside wage rate does not fall below a certain minimum level, given by $\omega\left(S_{0}\right)=\left[\frac{\gamma \alpha^{\alpha} S_{0}{ }^{\beta}}{G\left(S_{0}\right)^{1-\alpha}}\right]^{1 / \alpha}$, the outside employment opportunity absorbs what would otherwise be excessive effort $\left(\bar{E}-E^{*}\right)$ in the resource sector, thereby ensuring both full employment and sustainability of resource use. The required minimum outside wage rate depends on the initial resource stock, $S_{0}$, the elasticities of the harvest rate with respect to effort and the resource stock, $\alpha$ and $\beta$, the efficiency of the harvesting technology, $\gamma$, and the resource's biological characteristics as reflected by its natural (intrinsic) growth rate, $r$, and carrying capacity, $k$, which influence $G(S)$. Noting that $\lim _{S \rightarrow 0} \omega(S)=\infty^{18}$, it follows that when the initial resource stock is very small (for example, because economic measures to encourage resource conservation have been long neglected), then very high outside wage rates are needed to induce the RRB community to move to a sustainable path of resource use. It is also easy to verify that $\partial \omega\left(S_{0}\right) / \partial \gamma>0, \partial \omega\left(S_{0}\right) / \partial r<0$, and $\partial \omega\left(S_{0}\right) / \partial k<0$, implying respectively that the required minimum outside wage rate will be higher, the greater is the efficiency of harvesting technology, but the lower are the resource's intrinsic growth rate or carrying capacity.

\footnotetext{
${ }^{18}$ This follows from

$\lim _{S \rightarrow 0}\left[\omega(S)^{\alpha /(1-\alpha)} /\left(\alpha \gamma^{1 / \alpha}\right)\right]=\lim _{S \rightarrow 0}\left[S^{\beta /(1-\alpha)} / G(S)\right]=\lim _{S \rightarrow 0}[\beta /(1-\alpha)] S^{(\alpha+\beta-1) /(1-\alpha)} / r=\infty$.
} 


\subsection{Economic Diversification}

Next, we consider the option of economic diversification by creating a new economic activity; for example, a formal manufacturing sector. This sector produces a homogenous good according to production function $F(K, L)$, and accumulates capital according to

$$
\dot{K}=F(K, L)-c \geq 0, c \geq 0, K(0)=K_{0} \geq 0 \text { given }{ }^{19}
$$

where $K \geq 0$ is capital stock, $L \geq 0$ is labor input, and $c \geq 0$ is the consumption of the good expressed in units of harvest equivalent ${ }^{20}$. Thus, the community's instantaneous welfare can be written as $W(H+c, E+L)$, where, as usual, $H=H(E, S)$ is harvest and $E$ is labor input into the resource extraction sector.

To simplify the analysis and make the results comparable with the case of outside employment opportunity analyzed in the previous subsection, we assume that the production function has a form of $F(K, L)=f(K) L$, where $f$ is an increasing function $\left(f^{\prime}(K)>0\right)$. This implies that the marginal and average products of labor are equal to $f(K)$ and increase over time as capital is accumulated.

By the same argument as in the previous subsection, an optimal path entails full employment, i.e., $E+L=\bar{E}$ for all $t \geq 0$. Thus, the social planner's problem is

$$
\begin{gathered}
\max _{c(t), E(t)} \int_{0}^{\infty} W[H(E(t), S(t))+c(t), \bar{E}] e^{-\rho t} d t, \\
\text { subject to } \dot{S}(t)=G[S(t)]-H[E(t), S(t)], \\
\dot{K}(t)=F[K(t), \bar{E}-E(t)]-c(t) \\
0 \leq E(t) \leq \bar{E}, 0 \leq S(t), \quad \forall t \geq 0 ; \\
S(0)=S_{0} \geq 0, \quad K(0)=K_{0} \geq 0 \text { given. }
\end{gathered}
$$

Assume that this problem has a solution $\left(E^{*}(t), c^{*}(t), S^{*}(t), K^{*}(t)\right)$. Then, let us fix the path of capital stock at its optimal path, $K^{*}(t)$, and consider the partial problem:

\footnotetext{
${ }^{19}$ Note that we are implicitly assuming that investment in the industry sector is irreversible. We are also assuming that the initial capital stock, $K_{0}$, is given to the community either as a capital grant from the government or as foreign aid.

${ }^{20}$ Alternatively, we can assume the manufactured good and the resource harvest are perfect substitutes in consumption. Farm raised fish and harvested wild fish present an example.
} 


$$
\begin{gathered}
\max _{c(t), E(t)} \int_{0}^{\infty} W[H(E(t), S(t))+c(t), \bar{E}] e^{-\rho t} d t, \\
\text { subject to } \dot{S}(t)=G[S(t)]-H[E(t), S(t)], \\
\dot{K}^{*}(t)=F\left[K^{*}(t), \bar{E}-E(t)\right]-c(t) \\
0 \leq E(t) \leq \bar{E}, 0 \leq S(t), \forall t \geq 0 ; \\
S(0)=S_{0} \geq 0 \text { given. }
\end{gathered}
$$

Obviously, $\left(E^{*}(t), c^{*}(t), S^{*}(t)\right)$ is also a solution of this partial problem. We can now reduce the number of state variables in this problem to one by noting from the second constraint in (19') that

$$
c(t)=c(L(t), t)=f\left[K^{*}(t)\right] L(t)-\dot{K}^{*}(t), \quad L(t)=\bar{E}-E(t)
$$

and substituting from (20) in (19') to rewrite (19') as:

$$
\begin{gathered}
\max _{E(t)} \int_{0}^{\infty} W[H(E(t), S(t))+c(\bar{E}-E(t), t), \bar{E}] e^{-\rho t} d t, \\
\text { subject to } \dot{S}(t)=G[S(t)]-H[E(t), S(t)], \\
0 \leq E(t) \leq \bar{E}, 0 \leq S(t) \text { each } t \geq 0 ; \\
S(0)=S_{0} \geq 0 \text { given. }
\end{gathered}
$$

Define the associated Hamiltonian by

$$
\Psi(E, S, \lambda)=w^{D}(E, S, t)+\lambda[G(S)-H(E, S)]
$$

where $w^{D}(E, S, t)=[H(E, S)+c(\bar{E}-E, t)]^{\eta_{C}} \bar{E}^{\eta_{E}}$ is the social welfare function under economic diversification. As in the previous case, suppose $E^{*}>0$. Then, by the maximum condition we must have $\Psi_{E}\left(E^{*}, S^{*}, \lambda^{*}\right) \geq 0$, which, noting from (20) that $c_{L}\left(\bar{E}-E^{*}, t\right)=f\left(K^{*}(t)\right)$, implies

$$
\begin{aligned}
& H_{E}\left(E^{*}, S^{*}\right)-c_{L}\left(\bar{E}-E^{*}, t\right)=H_{E}\left(E^{*}, S^{*}\right)-f\left(K^{*}(t)\right) \\
& \geq\left(\eta_{C} \bar{E}^{\eta_{E}}\right)^{-1} \lambda H_{E}\left(E^{*}, S^{*}\right)\left(H\left(E^{*}, S^{*}\right)+c\left(\bar{E}-E^{*}, t\right)\right)^{1-\eta_{C}}>0 .
\end{aligned}
$$

Interestingly, condition (22) is essentially the same as condition (15) of the previous case, with $f\left(K^{*}(t)\right)$, the marginal product of labor, now corresponding to $\omega$, the outside wage rate, in (15) and (16). Thus, similar to Proposition 2, we have the following result: 
Proposition 3: If $f\left(K_{0}\right) \geq \omega\left(S_{0}\right)$, then $S^{*}(t) \geq S_{0}$, i.e. the resource stock never declines below the initial stock level on an optimal path.

Proof: Since $\dot{K} \geq 0, f\left(K^{*}(t)\right) \geq f\left(K_{0}\right) \geq \omega\left(S_{0}\right)$. So, if $S^{*}(t)=S_{0}$, then $\dot{S}^{*}(t) \geq 0$

Accordingly, economic diversification can lead the community to a sustainable full employment path as long as the initial grant of capital is not less than a certain minimum level, specifically $K_{0} \geq f^{-1}\left[\omega\left(S_{0}\right)\right]$ where, as before, $\omega\left(S_{0}\right)=\left[\frac{\gamma \alpha^{\alpha} S_{0}{ }^{\beta}}{G\left(S_{0}\right)^{1-\alpha}}\right]^{1 / \alpha}$.

The economic intuition for this result is similar to that of Proposition 2, once we note that in the present case the manufacturing wage rate, which is determined by $\omega(t)=f\left(K^{*}(t)\right)$ along the optimal path, plays the same role as the outside wage rate $\omega$ in the previous case (see (16)). Since the manufacturing wage rate increases over time as capital stock is accumulated, an initial stock of capital that meets the minimum required level ensures that along the optimal path the manufacturing wage rate is always sufficiently high to (optimally) absorb what would otherwise be excess effort in the resource sector. Since $f^{\prime}(K)>0$, the comparative statics results obtained in the previous case for $\omega\left(S_{0}\right)$ apply equally to the minimum required capital stock, $K_{0}=f^{-1}\left(\omega\left(S_{0}\right)\right)$. In particular, if the initial resource stock is very small, then a very large initial capital stock will be required to achieve a full employment sustainable path of resource use. This yields a general lesson for policymakers in the poor, resource-based developing countries; namely, the economic cost of achieving sustainability can significantly rise if appropriate remedial policies are not adopted early on. On the other hand, perhaps ironically, this result also shows that contrary to the view sometimes expressed by radical ecologists, industrialization and accumulation of manmade capital not only need not be at odds with the objective of resource sustainability, it can in fact facilitate it, at least in the context of RRB economies studied here.

\section{Conclusion}

In this paper we have focused on poor rural communities that depend on a renewable natural resource and provided an alternative explanation why such communities may choose a path of resource extraction that leads to extinction in a finite time. We have argued that in resource-based communities, being employed is a communal norm and as such a source of belonging and identity that confers socio-psychological benefits to members, and that the existence of these benefits as an integral part of the community's overall welfare can explain 
the community's strong desire (or even obsession) for full employment and its resistance to curbing the supply of "effort" (as frequently observed in fishing and forest communities). Embedding this argument into a standard bionomic model of optimal renewable resource extraction, we have shown that the communal norm of work combined with the lack of alternative income sources and the biological constraint on growth of the resource stock can interact to induce the community to choose a resource extraction path that results in resource extinction in finite time. Thus, our analysis offers an example of situations where the persistence of a social norm and benefits stemming from it (here the non-pecuniary benefits of employment) in the face of changing conditions (here the declining of the resource stock) can lead to a socially adverse outcome (here the resource extinction in a finite time).

Clearly, it is unrealistic to expect either the communal norm of full employment or the biological resource growth constraint to change in the short run. Therefore, one has to rely on economic policy to induce these communities towards a path of resource extraction that is both sustainable and compatible with the goal of full employment. We have shown that in principle successful economic policies would aim at providing the community with alternative employment sources either through employment opportunities outside the community (including labor migration) or by diversifying the community's economy by creating an indigenous capital-based economic activity. In either case, to be successful, the policies must ensure that the outside wage rate or the initial capital stock granted to the community do not fall below a certain minimum level, which depends on the existing size of the resource stock, on the characteristics of the community's harvesting technology, and on the biological growth characteristics of the resource in question.

No doubt the model developed in this paper highly simplified, thus suggesting several important directions for its extensions and future research. At a technical level, several possible extensions of the present model are noteworthy. For one thing, it would be interesting to relax the assumption of a constant population size and examine how the pressure of a growing population may exacerbate the problem of finite resource extinction and complicate the design of potential policies to avoid it. It would also be interesting to examine how the condition for finite time resource extinction would change if one departed from Cobb-Douglas specifications of the community's preferences and harvesting technology. Would, for example, greater elasticities of substitution between constituents of the community's well-being or between inputs in the harvesting technology lessen the possibility of resource extinction or accentuate it? How would they affect the policies to restore sustainability? Similarly, how would a more general specification of the social welfare function that differentiates the consumption of the resource from that of the manufactured consumption good, or a more general production function in the latter sector, affect our main conclusions? At a policy level, it would be interesting to consider a situation, perhaps not too unrealistic in the poor developing nations, in 
which no benevolent outside agency (public or private) may concern itself about the plight of possible resource extinction, so that the community has to rely on itself to come up with a remedial policy. One such policy could involve foregoing the full employment objective for some time and reducing the "effort" by a communal agreement to lay off some of the labor force, or shorten the length of individuals' activity period, in order to achieve a full employment sustainable path in the log run. What are the conditions for the community to reach such a binding agreement, given possible intergenerational conflicts of interests between employment and consumption? What would be the optimal shape of such an agreement (i.e., how much to reduce the effort at any time and for how long)? Also, it would be rewarding to explore socio-political and economic-organizational policies that could in the long run alter the underlying community's preferences or harvesting technology so as to avoid resource extinction. Finally, of particular value will be empirical research into the magnitude of the critical parameters constituting the condition for finite time resource extinction. 


\section{Appendix}

A1. Proof of Proposition 1

We first prove the following lemma:

Lemma 1: Assume that problem (2) has a solution for each $S \in[0, k]$. If $\Psi_{E E}(\tilde{E}(S, \lambda), S, \lambda)>0$ for all $S, \lambda>0$, the optimal path is full employment obsession path.

Proof: $\Psi_{E E}(\tilde{E}(S, \lambda), S, \lambda)>0$ implies that the maximum condition is satisfied at the corner; so, $E=0$ and/or $E=\bar{E}$. Thus, the optimal policy $E^{*}(S)$ for the problem (2) satisfies $E^{*}(S) \in\{0, \bar{E}\}$. Notice that this implies there is no optimal interior steady state. Let $\hat{S} \in[0, k]$ be the maximum stock level such that $E^{*}(S)=\bar{E}$ for all $S \in(\hat{S}, k]$. By Assumption (A3), an optimal path from $S_{0} \in[\hat{S}, k]$ decreases monotonically over time, but by definition of $\hat{S}$ it cannot fall below $\hat{S}$. Therefore $\hat{S}$ should be an optimal steady state. However $\hat{S}$ cannot be interior. $\hat{S}=k$ is obviously suboptimal. Thus, we conclude that $\hat{S}=0$. That is, $E^{*}(S)=\bar{E}$ for all $S \in(0, k]$

Proof of Proposition 1 :

Since $\quad \Psi_{E E}(\tilde{E}, S, \lambda)=w_{E E}(\tilde{E}, S)-\left(w_{E}(\tilde{E}, S) / H_{E}(\tilde{E}, S)\right) H_{E E}(\tilde{E}, S)$, $\Psi_{E E}(\tilde{E}(S, \lambda), S, \lambda)>0$ is equivalent to (6). Thus the statement is true by Lemma 1.

\section{A2. Finite Time Exhaustion}

Proposition A1: Let $\widehat{S}$ be the supremum such that if $0<S<\widehat{S}, \dot{S}=G(S)-H(\bar{E}, S)<0$. When initial stock $S_{0}$ is in $(0, \widehat{S})$, the path $S\left(t ; S_{0}\right)$ described by

$$
\dot{S}=G(S)-H(\bar{E}, S)=r S\left(1-\frac{S}{k}\right)-\gamma \bar{E}^{\alpha} S^{\beta}, \quad S(0)=S_{0},
$$

goes to zero within a finite time.

Proof: Notice that $\dot{S}=G(S)-H(\bar{E}, S)<r S-\gamma \bar{E}^{\alpha} S^{\beta}$. Let $\sigma\left(t, S_{0}\right)$ be the solution of $\dot{\sigma}=r \sigma-\gamma \bar{E}^{\alpha} \sigma^{\beta}, \sigma(0)=S_{0}$. This Bernoulli's differential equation is solved as 
$\sigma\left(t ; S_{0}\right)=\left[\left(S_{0}^{1-\beta}-\frac{\gamma \bar{E}^{\alpha}}{r}\right) e^{r(1-\beta) t}+\frac{\gamma \bar{E}^{\alpha}}{r}\right]^{\frac{1}{1-\beta}}$. From this, $\sigma\left(t ; S_{0}\right)$ goes to zero within a finite time if the initial stock satisfies $S_{0}{ }^{1-\beta}<\gamma \bar{E}^{\alpha} / r$. By construction, $S\left(t ; S_{0}\right)<\sigma\left(t ; S_{0}\right)$ and thus $S\left(t ; S_{0}\right)$ also goes to zero within a finite time. Note that since $\dot{S}\left(t ; S_{0}\right)<0, \lim _{t \rightarrow \infty} S\left(t ; S_{0}\right)=0$ is obvious, and we can arbitrarily choose an sufficiently small initial value.

Proposition A2: If $\gamma>\frac{r[(1-\beta) k]^{1-\beta}}{(\bar{E})^{\alpha}(2-\beta)^{2-\beta}}$, then $\dot{S}=G(S)-H(\bar{E}, S)<0$ for all $S \geq 0$.

Proof: Let $g(S ; \gamma)=r S(1-S / k)-\gamma \bar{E}^{\alpha} S^{\beta}$. If there is a unique pair $(\tilde{\gamma}, \tilde{S})$ such that $g(\tilde{S} ; \tilde{\gamma})=0, g_{S}(\tilde{S} ; \tilde{\gamma})=0$, and $g_{S S}(\tilde{S} ; \tilde{\gamma})<0$, then $g(S ; \gamma)<0$ for all $S>0$ and $\gamma>\tilde{\gamma}$. Solve the system of equations $g(\tilde{S} ; \tilde{\gamma})=0$ and $g_{S}(\tilde{S} ; \tilde{\gamma})=0$ and we have the unique solution

$$
\tilde{S}=\frac{1-\beta}{2-\beta} k, \tilde{\gamma}=\frac{r[(1-\beta) k]^{1-\beta}}{(\bar{E})^{\alpha}(2-\beta)^{2-\beta}} .
$$

It is easily seen that $g_{S S}(\tilde{S} ; \tilde{\gamma})=-r(2-\beta) / k<0$.

\section{A3. On the Existence of Optimal Paths}

We apply the Romer's Theorem (Romer, 1986) to prove the existence, because the theorem is applicable to a non-convex problem as in our model.

The reduced form welfare function for our model is written as:

$$
\omega(S, \dot{S})=\left\{\begin{array}{cc}
\gamma^{-\eta_{E} / \alpha}[G(S)-\dot{S}]^{\eta_{C}+\eta_{E} / \alpha} S^{-\eta_{E} \beta / \alpha} & \text { if } \dot{S} \in[G(S)-H(\bar{E}, S), G(S)], \\
-\infty & \text { otherwise. }
\end{array}\right.
$$

Since we have assumed $\eta_{E}-\alpha\left(1-\eta_{C}\right)>0, \omega$ is convex in $\dot{S}$. Hence, to apply the theorem, we introduce an "artificial" argument,

$$
\ddot{S}(t) \text { such that } \dot{S}(t)-\dot{S}(s) \equiv \int_{s}^{t} \ddot{S}(\tau) d \tau,
$$

and rewrite $\omega(S, \dot{S})$ as $\omega(S, \dot{S}, \ddot{S})$. Notice that in order for $\ddot{S}$ to exist almost everywhere, $\dot{S}$ must be absolutely continuous. This restriction is a cost of applying the Romer's Theorem. Another restriction is that we have to assume that $\ddot{S}$ is essentially bounded, i.e. there exists a real number $M$ such that ess.sup $|\ddot{S}(t)|<M$. The Romer's Theorem ensures the existence of 
optimal paths if the following two conditions are met:

(1) $\omega(S, \dot{S}, \ddot{S})$ is upper-semi continuous and $\omega(S, \dot{S}, \bullet)$ is concave for $\operatorname{all}(S, \dot{S}) \in \mathbb{R}^{2}$,

(2) There is a real number $m$ such that $\omega(S(t), \dot{S}(t), \ddot{S}(t)) \leq m-|\ddot{S}(t)|$ holds almost everywhere on $[0, \infty)$ and for all $(S(t), \dot{S}(t), \ddot{S}(t))$.

Our model obviously satisfies the first condition. For the second condition, notice that there is an upper bound of $\omega(S, \dot{S}, \ddot{S})$, say $\bar{\omega}(<\infty)$. Thus, choose $m=M+\bar{\omega}$ and then condition (2) is satisfied. 


\section{References}

Agerbo, E., T. Eriksson, B. Preben, and N. Westergard-Nielsen (1997), “Unemployment and Mental Disorders- an Empirical Analysis" University of Arhus, Arhus, Denmark.

Argyle, M. (2001), The Psychology of Happiness, New York: Taylor \&Francis.

Akerlof, G. (2000) "Economics and Identity”, Quarterly Journal of Economics, Vol. CXV, No. 3: 715-753.

Akerlof, G. A. and R. E. Kranton (2005), "Identity and the Economics of Organization", Journal of Economic Perspectives, Vol.19, No.1, Winter, 9-32.

Akerlof, G. A. and R. E. Kranton (2003), "Identity and the Economics of Organization", Working Paper, Department of Economics, University of Maryland, http://www.wam.umd.edu/ rkranton/identityandorganizations.pdf

Brander, J.A. and M.S. Taylor (1998),” The Simple Economics of Easter Island: A Ricardo-Malthus Model of Renewable Resource Use”, American Economic Review, Vol.88, No.1, PP: 119-138. Clark, A. E. (2003), "Unemployment as a Social Norm: Psychological Evidence from Panel Data", Journal of Labor Economics, Vol.21, No. 2, pp. 323-351

Clark, C. W. (1973), "Profit Maximization and the Extinction of Animal Species”, The Journal of Political Economy", Vol.81, No.4: 950-961

Clark, A. E., and A. J. Oswald (1994), "Unhappiness and Unemployment”, Economic Journal, Vol. 104, No. 424: 648-659

Cornes, R. and T. Sandler (1983), “On Commons and Tragedies”, American Economic Review, Vol.73, PP. 787-792

Cornes, R. and T. Sandler (1996), The Theory of Externalities, Public Goods and Club Goods, Second Edition, Cambridge University Press.

Darity, W. Jr. and A. H. Goldsmith (1996), "Social Psychology, Unemployment and Macroeconomics", Journal of Economic Perspective, Vol.10, No.1, winter, 121-140.

Di Tella, R., MacCulloch, R . J., and A. J. Oswald (2001), "Preferences Over Inflation and Unemployment: Evidence from Surveys of Happiness," American Economic Review, Vol. 91, No. 1, 335-341.

Di Tella, R., MacCulloch, R .J., and A. J. Oswald (2003), “The Macroeconomics of Happiness”, Review of Economics and Statistics, 85 (4), 809-827.

Dasgupta, P. (1982), The Control of Resources, Basil Blackwell, Oxford.

Dasgupta, P. and K. G. Maler (1995), 'Poverty, Institutions, and the Environmental Resource-Base", chapter 39 in Handbook of Development Economics, J. Behrman and T.N. Srinivasan, (eds.), Vol. IIIA, Elsevier Science, The Netherlands.

Farzin Y. H. (1984), "The Effect of Discount Rate on Depletion of Exhaustible Resources”, Journal of Political Economy, Vol. 92, No. 5, 841-851. 
Farzin, Y. H. and K. Akao (2004a), “ Non-pecuniary Value of Employment and Individual Labor Supply", Fondazione Eni Enrico Mattei WP 158.04.

Farzin, Y. H. and K. Akao (2004b), "Poverty, Non-pecuniary Value of Employment, and Natural Resource Extinction", mimeo, Department of Agricultural and Resource Economics, University of California, Davis.

Feather, N. T. (1990), The Psychological Impact of Unemployment. New York, Springer. Fryer, D. and R. Payne (1986), "Being Unemployed: A Review of the Literature on the Psychological Experience of Unemployment." In International Review of Industrial and Organizational Psychology, 1986, edited by C.L. Cooper and I. Robertson, pp.235-278, London, Wiley, 1986.

Frey, B. S. and A. and Stutzer (2002), "What Can Economists Learn From Happiness Research?" Journal of Economic Literature, Vol. XL, June, 402-435.

Gordon, J.R. (1954), “The Economic Theory of a Common Property Resource: The Fishery", Journal of Political Economy, Vol. 62, 1031-1039.

Hardin, G. (1968), "The Tragedy of Commons", Science 162, 1243-1247.

Jahoda, M. (1981), "Work, Employment and Unemployment: Values, Theories, and Approaches in Social Research”, American Psychologist, February, 36, 184-1991.

Jahoda, M. (1982), Employment and Unemployment: A Social Psychological Approach, Cambridge, Cambridge University Press.

Jahoda, M. (1988), "Economic Recession and Mental Health: Some Conceptual Issues", Journal of Social Issues, Fall, 44, 13-23.

Karpoff, J. M. (1985), "Nonpecuniary Benefits in Commercial Fishing: Empirical Findings from The Alaska Salmon Fisheries", Economic Inquiry, 23 (1), 159-174.

Korpi, T. (1997), "Is Well-Being Related to Employment Status? Unemployment, Labor Market Policies and Subjective Well-Being among Swedish Youth", Labor Economics, 4:2, 125-147.

Moffitt, R. (1983), “An Economic Model of Welfare Stigma”, American Economic Review, Vol. 73, No. 5, 1023-1035

Mulligan, C. B. (1998), "Pecuniary Incentives to Work in the United States During World War II", Journal of Political Economy, Vol.106, No. 5, 1033-1077.

Romer, P. (1986), “Cake Eating, Chattering, and Jumps: Existence Results for Variational Problems", Econometrica 54, 897-908.

Lucas, R.E., A.E. Clark, Y. Georgellis, and E. Diener, (2004), "Unemployment Alters The Set Point for Life Satisfaction", Psychological Science, Vol. 15, No. 1, PP. 8-13.

Spence, A.M. (1973), "Blue Whales and Applied Control Theory", in Systems Approaches to Environmental Problems, Bavarian Academy of Sciences, conference values, June 1973. 
Sorger, G. (1998) Markov-perfect Nash Equilibria in a Class of Resource Games, Journal of Economic Theory 11, 78-100.

Veblen, T. (1989), The Theory of the Leisure Class, New York, A.M. Kelly, 1975

Whelan, C. (1994), "Social Class, Unemployment and Psychological Distress", European Sociological Review 10, No.1, PP. 49-61.

Winelmann, L. and R. Winkelmann, (1998), "Why Are the Unemployed So Unhappy? Evidence from Panel Data", Economica, 65, No. 257, pp. 1-15. 